\title{
DEUTERON ELECTROMAGNETIC PROPERTIES WITH A POINCARÉ-COVARIANT CURRENT OPERATOR WITHIN FRONT-FORM HAMILTONIAN DYNAMICS
}

\author{
E. PACE \\ Dipartimento di Fisica, Università di Roma "Tor Vergata" and Istituto Nazionale \\ di Fisica Nucleare, Sezione Tor Vergata, Via della Ricerca Scientifica 1, I-00133 \\ Roma, Italy \\ G. SALMÈ \\ Istituto Nazionale di Fisica Nucleare, Sezione Roma I, P.le A. Moro 2, I-00185 \\ Roma, Italy
}

\begin{abstract}
Deuteron elastic and deep inelastic electromagnetic properties have been studied within the front-form Hamiltonian dynamics, using a Poincaré-covariant current operator. The deuteron elastic form factors are strongly sensitive to different realistic $N-N$ interactions, while the relevance of different nucleon form factor models is huge for $A\left(Q^{2}\right)$, weak for $B\left(Q^{2}\right)$ and negligible for the tensor polarization. The possibility to gain information on the neutron charge form factor from an analysis of $A\left(Q^{2}\right)$ has been investigated. The extraction of the neutron structure functions from the deuteron deep inelastic structure functions at high $x$ is largely affected by the use of our Poincaré-covariant relativistic approach instead of the usual impulse approximation within an instant-form approach.
\end{abstract}

\section{Introduction}

In Ref. 1 we have constructed an electromagnetic current operator for bound systems of interacting particles that satisfies Poincaré, parity and time-reversal covariance, together with Hermiticity and current conservation. This operator has been used to evaluate the deep inelastig structure functions (DISF) in an exactly solvable two-body model in Ref. $\mathrm{B}$, and the deuteron elastic form factors (ff) in Refs. 3.1 . Our current operator is obtained within the front-form Hamiltonian dynamics, starting from the free current in the Breit frame where initial and final three-momenta of the interacting system are directed along the spin quantization axis, $z$. In order to implement Hermiticity a term which implicitely introduces two-body terms in the current has to be considered. In the elastic case the continuity equation is automatically satisfied. By using this covariant current, one gets rid of the well-known ambiguities, which in the front-form approach plague the extraction of elastic and transition form factors for spin $\geq 1$ systems, when the free current is considered in the $q^{+}=0$ reference frame. Indeed, in our approach the results 

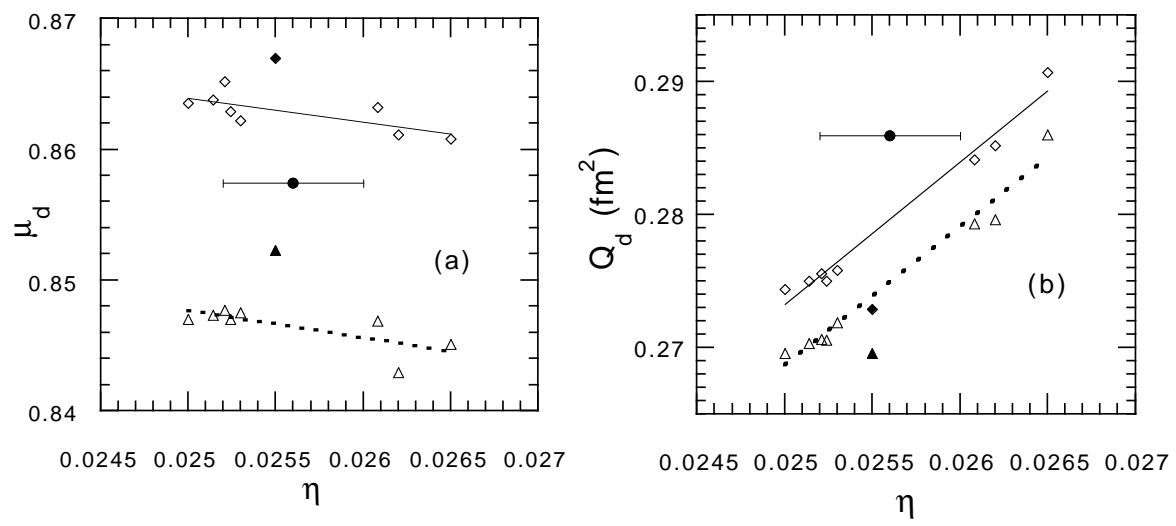

Figure 1. (a) Deuteron magnetic moment, $\mu_{d}$, vs the asymptotic normalization ratio $\eta=A_{D} / A_{S}$, for different $N-N$ interactions. The full dot represents the experimental values for $\mu_{d}$ and $\eta$; empty triangles and diamonds correspond to the nonrelativistic and relativistic results, respectively, while the solid and dashed lines are linear fits for these results. Full triangle and diamond are the results of the $C D-$ Bonn interaction. (b) The same as in (a), but for the deuteron quadrupole moment, $Q_{d}$ (After Ref. B)

for elastic and transition form factors, as well as for deep inelastic structure functions, are independent of the matrix elements of the current which are employed in the calculation, as it must be. Then the deuteron elastic ff and DISF can be safely calculated from front-form deuteron wave functions.

In this paper we report the results of our thorough investigation on the sensitivity of deuteron $\mathrm{ff}$ to different realistic $N-N$ interactions and to different nucleon form factor models $\mathrm{B}-\mathrm{B}$. Furthermore we report our studies on the extraction of the neutron structure function, $F_{2}^{n}(x)$, from the deuteron deep inelastic structure function $F^{D}(x)$ and on the relevance of our Poincarécovariant relativistic approach 10.12 .

\section{Deuteron Elastic Form Factors}

As a first application of our current operator for elastic electron scattering, we determined $\mathrm{B}$ the deuteron magnetic and quadrupole moments, $\mu_{d}$ and 

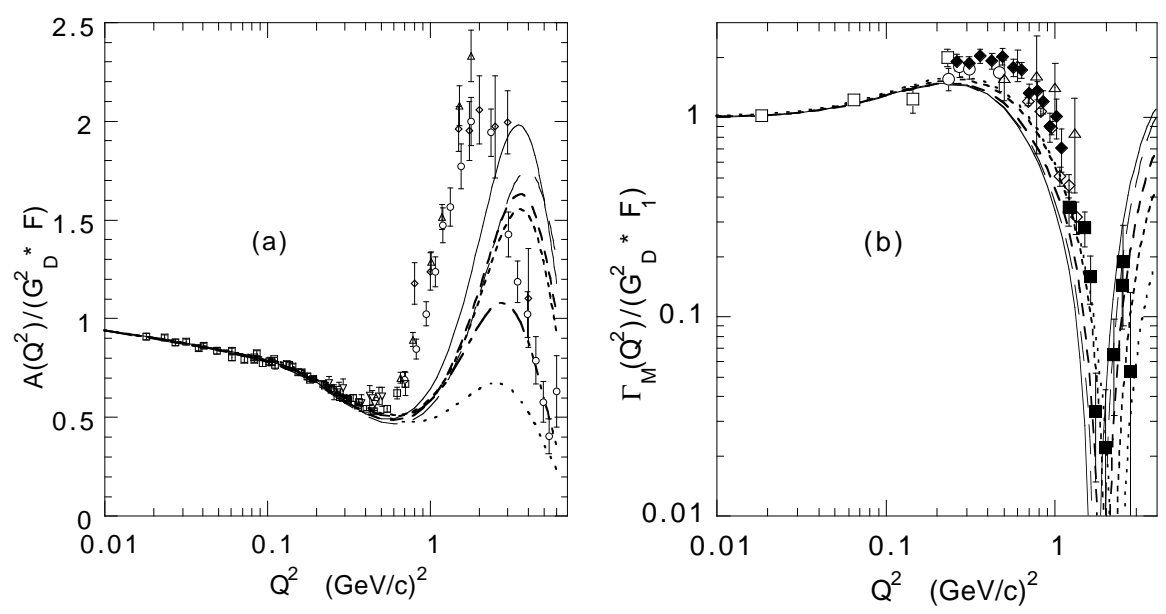

Figure 2. (a) The ratio $A\left(Q^{2}\right) /\left(G_{D}^{2} \cdot F\right)$ (with $G_{D}=\left(1+Q^{2} / 0.71\right)^{-2}$ and $\left.F=\left(1+Q^{2} / 0.1\right)^{-2.5}\right)$ vs $Q^{2}$ obtained with different $N-N$ interactions and the nucleon $\mathrm{ff}$ of Ref. 17 . Solid line: $R S C$ interaction; dashed line: $A V 18$ interaction; dot-dashed line: Nijmegen 1 interaction; long-dashed line: Nijmegen 2 interaction; short-dashed line: Nijmegen 93 interaction; dotted line: $C D-B o n n$ interaction. (b) As in Fig. 1(a), but for the reduced form factor $\Gamma_{M}\left(Q^{2}\right) /\left(G_{D}^{2} \cdot F_{1}\right)$ with $\Gamma_{M}\left(Q^{2}\right)=\left[G_{M}\left(Q^{2}\right) m_{p} /\left(\mu_{d} m_{d}\right)\right]^{2}$ and $F_{1}=\left(1+Q^{2} / 0.1\right)^{-3}$. The Nijmegen 1 result is verysimilar to the $C D-B o n n$ one and is not reported in this figure. (After Ref. 6 )

$Q_{d}$, by adopting front-form deuteron wave functions corresponding to a large set of realistic $N-N$ interactions (RSC, Paris 12 , Argonne $V 14$ 13 and V18 14, Nijmegen - 1, -2, -93 and Reid93 15, $C D-B o n n ~ 16)$. For both quantities a linear behaviour against the deuteron asymptotic normalization ratio $\eta=A_{D} / A_{S}$ was found (see Fig. 1). Therefore, the usual disagreement between theoretical and experimental results is largely removed and explicit contributions of dynamical two-body currents and isobar components in the deuteron should play a minor role at $Q^{2} \rightarrow 0$, using our Poincaré-covariant relativistic framework 3 .

In this section the deuteron electromagnetic form factors, obtained with our current operator, are analyzed in detail by studying the dependence on different realistic $N-N$ interactions and on different nucleon form factor 

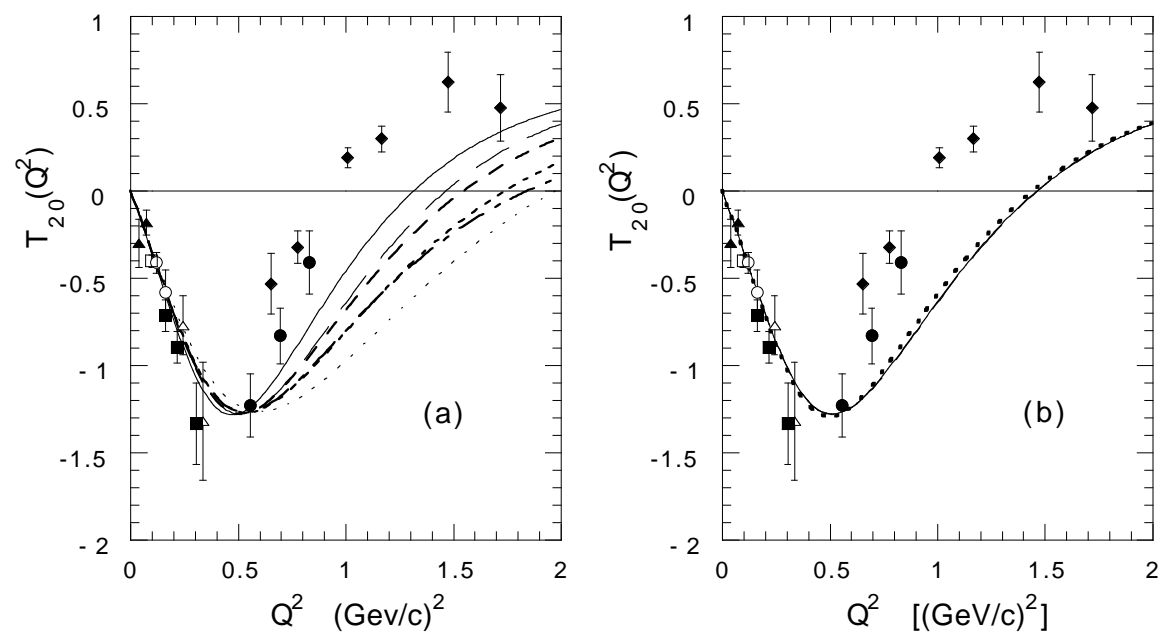

Figure 3. (a) The tensor polarizations $T_{20}\left(Q^{2}\right)$ obtained with different $N-N$ interactions and the nucleon $\mathrm{ff}$ of Ref. 17. The lines have the same meaning as in Fig. 2(a). (b) The tensor polarization $T_{20}\left(Q^{2}\right)$ obtained with the nucleon ff models of Ref. 18 (solid line), Ref. 19 (dotted line), and Ref. 17 (dashed line). (After Ref. 6 )

models, but, first of all, let us note the large differences between relativistic and non relativistic calculations, even at low momentum transfer values $\left(Q^{2} \simeq\right.$ $\left.0.2-0.3(\mathrm{GeV} / \mathrm{c})^{2}\right)$ - 1 .

As shown in Figs. 2, 3 and 4, different interactions yield huge effects on $A\left(Q^{2}\right)$ at $Q^{2} \geq 1(\mathrm{GeV} / \mathrm{c})^{2}$, while for $B\left(Q^{2}\right)$ and the tensor polarizations their effects are large already at $Q^{2} \geq 0.5(\mathrm{GeV} / \mathrm{c})^{2}$ (see also Ref. 4 ). In Fig. 2 a suitable reduced plot as been used for $A\left(Q^{2}\right)$ and $B\left(Q^{2}\right)$ to allow a closer comparison between theoretical results and experimental data. An interesting correlation between the S-state deuteron momentum distribution and the deuteron form factors has been found. Indeed, interactions which have essentially the same S-state momentum distribution, $n_{o}(k)$, as the $A V 18$ and Reid93 interactions, give the same results for $A\left(Q^{2}\right), B\left(Q^{2}\right)$ and the tensor polarizations (for this reason the curves corresponding to Reid93 are not reported in the figures). Furthermore, as shown in Fig. 5, the behaviour of $B\left(Q^{2}\right)$ and of the S-state deuteron momentum distribution for correspond- 

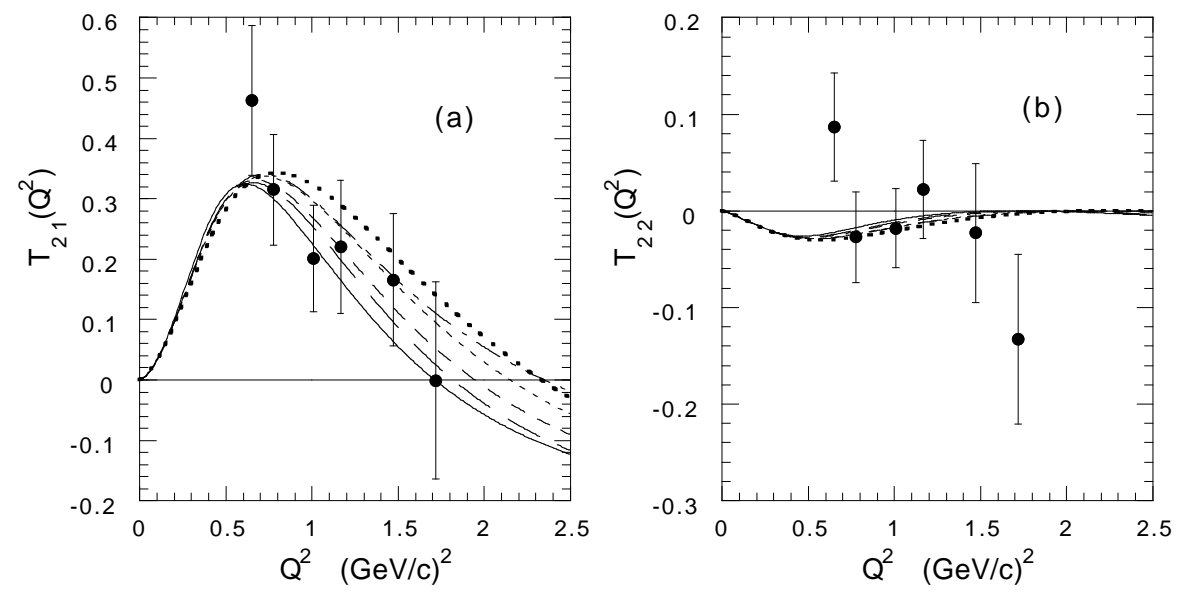

Figure 4. The tensor polarizations $T_{21}\left(Q^{2}\right)$ (a) and $T_{22}\left(Q^{2}\right)(\mathrm{b})$, obtained with different $N-N$ interactions and the nucleon $\mathrm{ff}$ of Ref. 17 . The lines have the same meaning as in Fig. 2(a). Experimental data are from Ref. 21 . (After Ref. 6)

ing interactions is similar. In order to perform the comparison between the deuteron $\mathrm{ff}$ and S-state momentum distribution on a quantitative basis, we report in Fig. 6 the position of the minimum of $B\left(Q^{2}\right)$ and the position of the second zero of $T_{20}\left(Q^{2}\right)$ against the nonrelativistic S-state kinetic energy, $T_{S}$. A distinct linear behaviour has been found: an higher value of $T_{S}$ moves the minimum of $B\left(Q^{2}\right)$ and the second zero of $T_{20}\left(Q^{2}\right)$ to a lower momentum transfer $6-6$.

Within an analysis which neglects two-body currents and isobar components, this behaviour suggests the possibility of a description of the experimental data for $T_{20}\left(Q^{2}\right)$ with interactions exhibiting high values of $T_{\mathrm{m}}$. Let us note that recent measurements of the $S-D$ mixing parameter, $\epsilon_{1} 20$, point to a stronger tensor force than the one exhibited by the interaction models we have analyzed. In turn, a stronger tensor force is favoured by an high degree of locality, which yields significantly larger kinetic energies and, in particular, larger values of $T_{S} 22$. Then, one can argue that a $N-N$ interaction, able to reproduce the recent measurements of $\epsilon_{1}$, could yield agreement between experimental and theoretical values for $T_{20}\left(Q^{2}\right)$ and a minimum for $B\left(Q^{2}\right)$ 

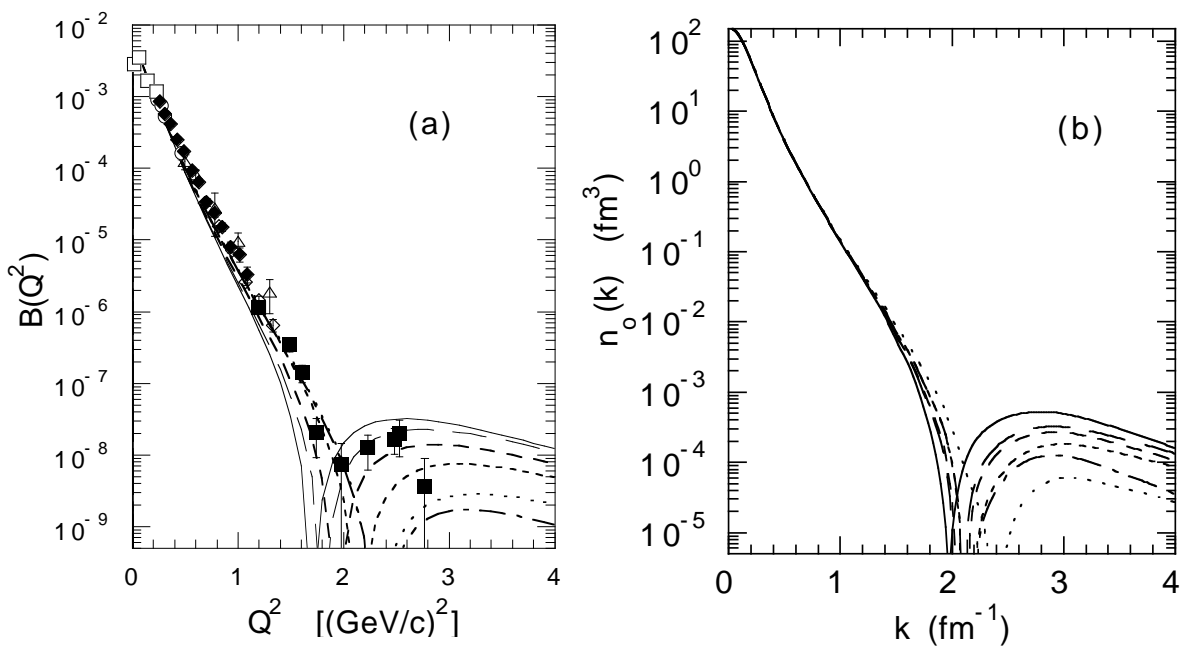

Figure 5. (a) The deuteron ff $B\left(Q^{2}\right)$ obtained with different $N-N$ interactions and the nucleon ff of Ref. 17 . The lines have the same meaning as in Fig. 2(a). (After Ref. G) (b) The S-state deuteron momentum distribution corresponding to different $N-N$ interactions. The lines have the same meaning as in Fig. 2(a).

slightly lower than the value indicated by the available experimental data. Therefore, if the new, more precise, measurement of $B\left(Q^{2}\right)$ which is planned at TJNAF will show a lower value for the position of the minimum, both $T_{20}\left(Q^{2}\right)$ and $B\left(Q^{2}\right)$ could be reproduced, without a relevant role of dynamical two-body currents and isobar components.

Different nucleon $\mathrm{ff}$ models have very large effects on $A\left(Q^{2}\right)$, small effects on $B\left(Q^{2}\right)$ and negligible effects on the tensor polarizations $T_{20}\left(Q^{2}\right), T_{21}\left(Q^{2}\right)$, $T_{22}\left(Q^{2}\right)$ (see Figs. 3(b), 7(a) and Ref. 4 ). In view of the large uncertainties in the experimental knowledge of the neutron charge ff, we have tried to find a phenomenological description for the nucleon charge $\mathrm{ff}$, able to describe the deuteron elastic data for $A\left(Q^{2}\right)$ and, at the same time, to reproduce the recent data for the ratio $G_{E}^{p} / G_{M}^{p}$ 23 The proton and neutron magnetic ff have been fixed to the model of Ref. 19 and the parametrization of the same model has been used for the proton and neutron charge ff, but changing the values of the parameters. As shown by the solid and long-dashed lines in Fig. 

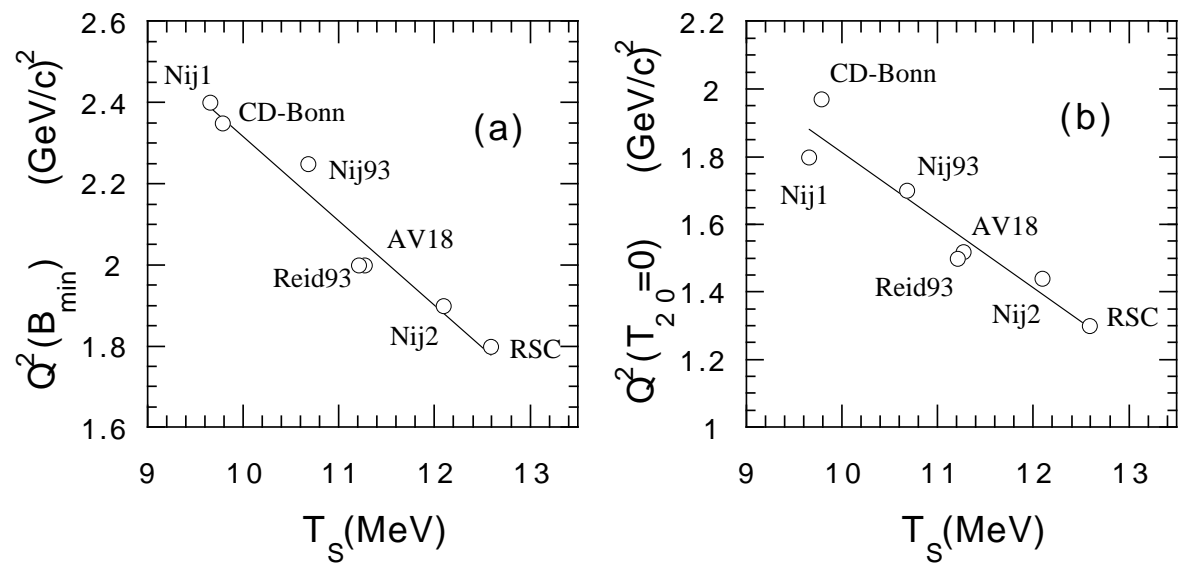

Figure 6. (a) The position of the minimum of $B\left(Q^{2}\right)$, and (b) the position of the second zero of $T_{20}\left(Q^{2}\right)$, corresponding to the nucleon $\mathrm{ff}$ of Ref. $\mathrm{E}$, vs the nonrelativistic S-state kinetic energy of the deuteron for different realistic interactions. The experimental values of $Q^{2}\left(B_{\min }\right)$ and $Q^{2}\left(T_{20}=0\right)$ are $\simeq 1.8$ $(\mathrm{GeV} / \mathrm{c})^{2}$ and $\simeq 1.0(\mathrm{GeV} / \mathrm{c})^{2}$, respectively. (After Ref. 4 )

7(a), corresponding to the $R S C$ and Reid93 interactions, respectively, an excellent agreement with the experimental data for $A\left(Q^{2}\right)$ can be obtained, except for small differences in the region around $Q^{2}=1(\mathrm{GeV} / \mathrm{c})^{2}$. The obtained $G_{E}^{p}\left(Q^{2}\right)$ goes exactly through the data of Ref. 23, while $G_{E}^{n}\left(Q^{2}\right)$ turns out to be somewhat higher than the Gari-Krümpelmann model around $Q^{2}=0.8(\mathrm{GeV} / \mathrm{c})^{2}$ and smaller beyond. Similar results can also be obtained using other realistic interactions. Changing the $N-N$ interaction a range of possible values for $G_{E}^{n}\left(Q^{2}\right)$ could be identified.

However, these results on $G_{E}^{n}\left(Q^{2}\right)$ cannot be taken too seriously. Indeed, the contributions of explicit two-body currents and of isobar components in the deuteron wave function have to be thoroughly investigated, before drawing definite conclusions. 

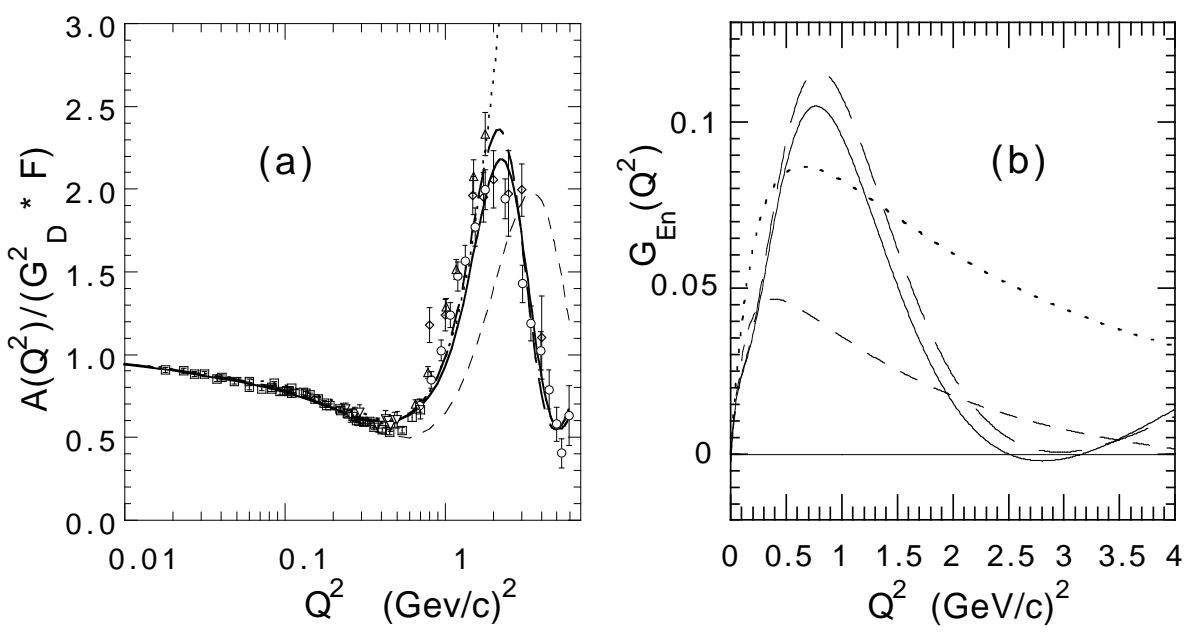

Figure 7. (a) Solid and long-dashed lines: the ratio $A\left(Q^{2}\right) /\left(G_{D}^{2} \cdot F\right)$ calculated with $R S C$ and Reid93 interactions, respectively, and fitted nucleon charge ff (see text); dotted and short-dashed lines: the ame as the long-dashed line, but with the nucleon ff of Ref. 19 and Ref. 17 , respectively. (After Ref. G) (b) The neutron charge form factor, $G_{E}^{n}\left(Q^{2}\right)$. Solid and long-dashed lines are the fitted ff, corresponding to the $R S C$ and Reid93 interactions, respectively; dotted and short-dashed lines are the neutron charge ff of Ref. 19 and Ref. 17, respectively.

\section{Deuteron Deep Inelastic Structure Function}

The Poincaré-covariant current operator can also be applied to the deep inelastic electron scattering off nuclei 211 . In convolution models 24 the deuteron DISF, in the Bjorken limit, is

$$
F_{2}^{D}(x)=\int_{x}^{M_{D} / M}\left[F_{2}^{p}(x / z)+F_{2}^{n}(x / z)\right] f^{D}(z) d z
$$

where $x=Q^{2} /(2 M \nu)$, while $M$ and $M_{D}$ are the nucleon and deuteron masses, respectively. The distribution $f^{D}(z)$ describes the structure of the deuteron 

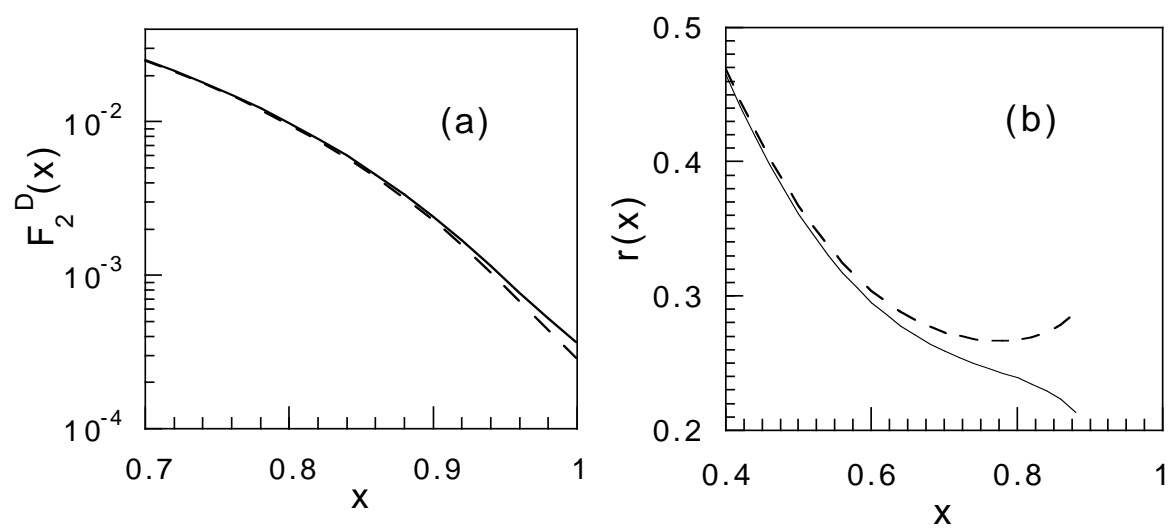

Figure 8. (a) The deuteron DISF, $F_{2}^{D}(x)$, obtained from Eq. (1) using the $A V 18 N-N$ interaction and (b) the ratio $r(x)$ of neutron to proton DISF, extracted by the recurrence relation (Eq. (仿) from simulated data for the deuteron DISF (see text). The dashed and solid lines are obtained using in the denominator of Eq. (4) the expressions of Eqs. (2) or (3) for the distribution $f^{D}(z)$, corresponding to the impulse approximation within the instant-form approach or to our Poincaré-covariant current operator, respectively.

system. In the usual approach 25.26, which makes use of the impulse approximation within an instant-form framework, the distribution $f^{D}(z)$ has the following expression

$$
f_{I F}^{D}(z)=\int d \vec{p} n^{D}(|\vec{p}|) \delta\left(z-\frac{p q}{M \nu}\right) z C
$$

where $n^{D}(|\vec{p}|)$ is the nucleon momentum distribution in the deuteron, $C$ a normalization factor, $q \equiv(\nu, \vec{q})$ the four-momentum transfer, and $p$ the four-momentum of an off-mass shell nucleon, i.e., $p \equiv\left(p^{0}, \vec{p}\right)$ with $p^{0}=$ $M_{D}-\sqrt{M^{2}+|\vec{p}|^{2}}$. Within the front-form Hamiltonian dynamics, using the Poincaré-covariant current operator, the expression for the deuteron DISF is again given by Eq. (11), but the distribution $f^{D}(z)$ reads as follows 11 (see also Ref. 24):

$$
f_{F F}^{D}(z)=\int d \vec{k} n^{D}(|\vec{k}|) \delta\left(z-\xi \frac{M_{D}}{M}\right)
$$


where $\xi=p^{+} / P^{+}=\left[k_{z}+\sqrt{M^{2}+|\vec{k}|^{2}}\right] /\left[2 \sqrt{M^{2}+|\vec{k}|^{2}}\right]$. It has to be stressed that, at variance with the instant-form dynamics, in the front-form Hamiltonian dynamics the nucleons are always on their mass shell, i.e. $p^{2}=M^{2}$. In Fig. 8(a) we report the deuteron DISF obtained with the nucleon momentum distribution corresponding to the $A V 18 N-N$ interaction, using the model of Ref. 27 for the nucleon DISF. The difference between the results obtained with the two-different expressions for $f^{D}(z)$ is very small, except at $x \sim 1$.

In Ref. 1 it has been proposed to extract the ratio $r(x)=F_{2}^{n}(x) / F_{2}^{p}(x)$ from the experimental data for the deuteron DISF, $F_{2}^{\text {Dexp }}(x)$, by the following recurrence relation :

$$
r^{(n+1)}(x)=\frac{F_{2}^{\operatorname{Dexp}}(x)\left[1+r^{(n)}(x)\right]}{\int_{x}^{M_{D} / M}\left[1+r^{(n)}(x / z)\right] F_{2}^{p}(x / z) f^{D}(z) d z}-1
$$

It was shown 10 that a rapid convergence can be obtained, largely independent on the assumed zero-order approximation, $r^{(0)}(x)$, up to $x \leq 0.90$.

In order to investigate the sensitivity of the extraction of $r(x)$ to different expressions for $f^{D}(z)$ (cf. Eq. (2) and (3)), we have extracted $r(x)$ by simulating the data of the deuteron DISF, $F_{2}^{D e x p}$, through a theoretical evaluation. As a matter of fact, the deuteron DISF has been calculated by Eq. (1) using for $f^{D}(z)$ the average of $f_{F F}^{D}(z)$ and $f_{I F}^{D}(z)$, corresponding to the $A V 18 N-N$ interaction and the model of Ref. 27 for the nucleon DISF. Then, Eq. (1) has been applied, by inserting in the denominator $f_{F F}^{D}(z)$ or $f_{I F}^{D}(z)$, alternatively, always corresponding to the $A V 18$ interaction. As shown in Fig. 8(b), the differences in the extracted functions $r(x)$ become quite sizeable at $x \geq 0.7$. Moreover we have shown that the differences in the extracted values of $r(x)$ are of the same order if $f_{F F}^{D}(z)$ or $f_{I F}^{D}(z)$ are used to generate the simulated data, instead of their average value. Therefore a Poincaré-covariant treatment of relativity appears to be important in the extraction of the neutron deep inelastic structure function at high $x$. An analysis of the experimental data for $F_{2}^{D}$ will be performed in Ref. 11 .

\section{Conclusions}

Deuteron elastic form factors and deep inelastic structure functions have been studied within the front-form Hamiltonian dynamics, using a Poincarécovariant current operator for bound systems of interacting particles, which satisfies parity and time-reversal covariance, as well as Hermiticity and current conservation. The current is built up from the free one in the Breit reference frame where three-momentum transfer of the interacting system is directed

Cort2000: submitted to World Scientific on November 14, 2018 
along the spin quantization axis, $z$. This current allows one to obtain elastic and transition form factors, as well as deep inelastic structure functions, with no ambiguity.

The Poincaré-covariant relativistic approach has been shown to have a big impact with respect to nonrelativistic calculations, both in the elastic and deep inelastic case and even at low momentum transfer values.

As far as the elastic ff are concerned, the usual disagreement between theoretical and experimental values for $\mu_{d}$ and $Q_{d}$ is largely removed and therefore contributions beyond impulse approximation are expected to play a minor role at $Q^{2} \rightarrow 0$. Large effects have been found from different nucleon form factor models and different realistic $N-N$ interactions. It is especially noteworthy an interesting correlation between the deuteron S-state kinetic energy, $T_{S}$, and the positions of the minimum of $B\left(Q^{2}\right)$ and of the second zero of $T_{20}\left(Q^{2}\right)$.

We stress that, when comparing the theoretical results of different approaches with the experimental data for the deuteron $\mathrm{ff}$, all the observables should be considered at the same time, before drawing definite conclusions on the quality of the various approaches. Furthermore, the possible different $N-N$ interactions and nucleon ff models should be carefully investigated.

Our next step will be to study within our approach the role of contributions from explicit two-body current and from isobar configuration in the deuteron wave function.

As far as the deep inelastic structure function is concerned, the relevance of a Poincaré-covariant relativistic approach has been clearly shown in the extraction of the neutron DISF from the deuteron DISF. Even if the effects in the calculation of $F_{2}^{D}(x)$ are small, the consequences of Poincaré-covariance in the extraction of the neutron DISF become important at high $x$, especially in view of the experiments planned at TJNAF for measuring $F_{2}^{n}(x)$ up to $x=0.8328$.

\section{Acknowledgments}

The authors are highly indebted to S. Scopetta for his kind help in obtaining the deuteron deep inelastic structure function and in the extraction of the neutron deep inelastic structure function.

\section{References}

1. F.M. Lev, E. Pace, G. Salmè, Nucl. Phys. A 641, 229 (1998).

2. E. Pace, G. Salmè, F.M. Lev, Phys. Rev. C 58, 2655 (1998). 
3. F.M. Lev, E. Pace, G. Salmè, Phys. Rev. Lett. 83, 5250 (1999).

4. F.M. Lev, E. Pace, G. Salmè, Phys. Rev. C 62, 064004 (2000).

5. F.M. Lev, E. Pace, G. Salmè, Few-Body Systems, Suppl. 10, 135 (1999).

6. F.M. Lev, E. Pace, G. Salmè, Nucl. Phys. A 663\&664, 365c (2000).

7. G. Salmè, F.M. Lev, E. Pace, Few-Body Systems, Suppl. 12, 235 (2000).

8. E. Pace and G. Salmè, in Proceedings of the XVI International Conference on Few-Body Problems in Physics, March 6-10, 2000, Taipei (Taiwan), to appear in Nucl. Phys. A.

9. E. Pace and G. Salmè, in Proceedings of the XVII European Few-Body Conference, September 10-17, 2000, Evora (Portugal), to appear in Nucl. Phys. A.

10. E. Pace, G. Salmè, and S. Scopetta, in Proceedings of the XVII European Few-Body Conference, September 10-17, 2000, Evora (Portugal), to appear in Nucl. Phys. A, nucl-th/0009028; E. Pace, G. Salmè, and S. Scopetta, to be published.

11. E. Pace, G. Salmè, to be published.

12. M. Lacombe et al., Phys. Rev. C 21, 861 (1980).

13. R.B. Wiringa, R.A. Smith and T.A. Ainsworth, Phys. Rev. C 29, 1207 (1984).

14. R.B. Wiringa, V.G.J. Stoks and R. Schiavilla, Phys. Rev. C 51, 38 (1995).

15. V.G.J. Stoks, R.A.M. Klomp, C.P.F. Terheggen, J.J. de Swart, Phys. Rev. C 49, 2950 (1994).

16. R. Machleidt, F. Sammarruca, Y. Song, Phys. Rev. C 53, R1483 (1996).

17. G. Höhler et al., Nucl. Phys. B 114, 505 (1975).

18. P. Mergell, U-G. Meissner, and D. Drechsel, Nucl. Phys. A 596, 367 (1996).

19. M. Gari and W. Krümpelmann, Z. Phys. 322, 689 (1985).

20. B.W. Raichle et al., Phys. Rev. Lett. 83, 2711 (1999).

21. D. Abbott et al., Phys. Rev. Lett. 84, 5053 (2000).

22. A. Polls et al., Phys. Lett. B 432, 1 (1998).

23. M.K. Jones et al., Phys. Rev. Lett. 84, 1398 (2000).

24. U. Oelfke, P.U. Sauer, F. Coester, Nucl. Phys. A 518, 593 (1990).

25. C. Ciofi degli Atti and S. Liuti, Phys. Rev. C 41, 1100 (1990); Phys. Rev. C 44, 1269 (1991).

26. C. Ciofi degli Atti, S. Scopetta, E. Pace, G. Salmè, Phys. Rev. C 48, R968 (1993).

27. J. J. Aubert et al., Nucl. Phys. B 293, 740 (1987).

28. I.R. Afnan, F. Bissey, J. Gomez, A.T. Katramatou, W. Melnitchouk, G.G. Petratos, A.W. Thomas, Phys. Lett. B 493, 36 (2000). 